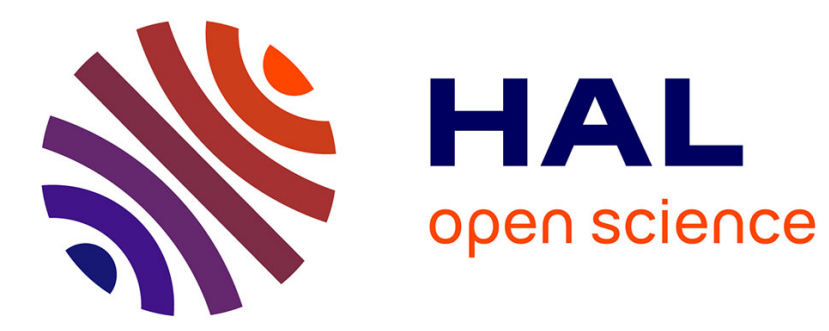

\title{
Vortex ring head-on collision with a heated vertical plate
}

Gabriel Arévalo, Rodrigo H. Hernández, Christian Nicot, Frédéric Plaza

\section{To cite this version:}

Gabriel Arévalo, Rodrigo H. Hernández, Christian Nicot, Frédéric Plaza. Vortex ring head-on collision with a heated vertical plate. Physics of Fluids, 2007, 19, pp.083603. 10.1063/1.2759880 . hal00272153

\section{HAL Id: hal-00272153 \\ https://hal.science/hal-00272153}

Submitted on 15 Jun 2012

HAL is a multi-disciplinary open access archive for the deposit and dissemination of scientific research documents, whether they are published or not. The documents may come from teaching and research institutions in France or abroad, or from public or private research centers.
L'archive ouverte pluridisciplinaire HAL, est destinée au dépôt et à la diffusion de documents scientifiques de niveau recherche, publiés ou non, émanant des établissements d'enseignement et de recherche français ou étrangers, des laboratoires publics ou privés. 


\title{
Vortex ring head-on collision with a heated vertical plate
}

\author{
G. Arévalo and R. H. Hernández ${ }^{\text {a) }}$ \\ LEAF-NL, Departamento de Ingeniería Mecánica, Universidad de Chile, Casilla 2777, Santiago, Chile \\ C. Nicot and F. Plaza \\ Laboratoire de Mecanique des Fluides et d'Acoustique, CNRS Université de Lyon-Ecole Centrale de Lyon- \\ Université Lyon I-INSA Lyon E.C.L., 36 avenue Guy de Collongue, F-69134 Ecully, France
}

(Received 22 December 2006; accepted 5 June 2007; published online 21 August 2007)

\begin{abstract}
We report experimental results of the normal impact of a vortex ring in air on a vertical heated plate at constant temperature. We address the case in which the natural convection boundary layer is laminar and the vortex ring is stable. Vortex rings are created by pushing air through a circular exit orifice of a cavity, using a piston-cylinder system. The impinging vortex ring perturbs both the thermal and dynamical boundary layers where we measure the total heat flux exchanged by the heated plate and visualize the vortex motion during the impact. This unsteady impingement process is investigated for different vortex sizes and self-induced velocities, characterized by the Reynolds number of the ring. As a result, a localized heat transfer enhancement is originated by the ring impingement, which increases with the Reynolds number. (C) 2007 American Institute of Physics. [DOI: $10.1063 / 1.2759880]$
\end{abstract}

\section{INTRODUCTION}

Vortex structures are known to play an important role in convective heat transfer enhancement. Indeed, common tricks to increase wall heat transfer consist in placing specific obstacles, called vortex promoters, upstream a heated wall. These obstacles are supposed to create horseshoe vortices that interact with the thermal boundary layers. ${ }^{1}$ Evidence of heat transfer enhancement is given in a significant mean heat flux increase, but a detailed scenario of this mechanism, based on experimental observation, is still lacking. To give a clue on this problem, we address the case of a well studied vortical structure, the vortex ring. Although different from horseshoe vortices, there are various reasons for this choice: vortex rings are easy to create, reproducible, and well-documented; ${ }^{2}$ their production can be totally independent of the boundary layer flow close to the wall, which allows their characteristic parameters to change freely; as impinging jets are efficient for heat transfer enhancement, ${ }^{3}$ in particular in microsystems, it could be profitable to examine the case of head-on vortex ring collision on a wall; finally, as mentioned in Ref. 2, vortex rings admit invariant states and are therefore candidates for elementary excitation in turbulence. ${ }^{4}$ As there is very poor agreement on the universality of heat transfer enhancement by incoming freestream turbulence, ${ }^{5}$ it is interesting to focus on what could be an elementary scenario for turbulence interaction with a heated wall. Vortex ring head-on collision with a wall, with neither thermal effects nor a mean flow (absence of boundary layer), has already been studied both experimentally and numerically. ${ }^{6,8,7,9}$ Depending on vortex ring velocity, the vortex can be staying on the wall, slightly increasing its diameter and vanishing by viscous dissipation. At higher initial

\footnotetext{
a) Author to whom correspondence should be addressed. Electronic mail: rohernan@ing.uchile.cl. URL: http://www.leafnl.uchile.cl
}

velocities, the ejection of secondary ring, normal to the wall, can be observed. ${ }^{7}$

In the present work, we report an experiment where a stable vortex ring ${ }^{10}$ collides on a vertical boundary layer generated by natural convection. The interest of this study is to understand what happens when the vortex meets a zone of high vorticity like a boundary layer. We study in detail the heat transfer enhancement occurring during the collision with an isothermal heated vertical surface, which also behaves as a heat flux sensor. We can link the phenomenological observations of the dynamical behavior of the ring with a quantitative measurement of the global heat flux released by the heated surface to the fluid. We focus particularly on the ability for the vortex ring to increase convective heat transfer. This paper is organized as follows. Section II describes the experimental setup, the properties of the ring produced in our experiment, and a characterization of the natural convection boundary layer. Results are presented in Sec. III. Concluding remarks are summarized in Sec. IV.

\section{EXPERIMENTAL SETUP AND FLOW PROPERTIES}

\section{A. General description}

Experiments were carried out at the LEAF-NL laboratory at the University of Chile. Figure 1 shows the complete experimental apparatus. A vortex ring of diameter $D$ and self-induced velocity $U_{a}$ is created in a still air chamber, itself in a closed room using a vortex ring generator made with a flat circular piston driven by a loudspeaker pushing an air-slug volume through the center hole of diameter $D_{0}$ of a smooth plexiglass disk $\left(D_{0}=13,15,17,20 \mathrm{~mm}\right.$ in this work). Different initial ring impulses are obtained by changing the rising time $\left(6.9<\tau_{r}<51 \mathrm{~ms}\right)$ of a forcing (constant peak amplitude) signal, $s(t)$, used to drive the speaker, where $\tau_{r}$ is defined as the time for the leading edge of the pulse $s(t)$ to rise from 0 to $63 \%$ of its peak value after a TTL trigger 


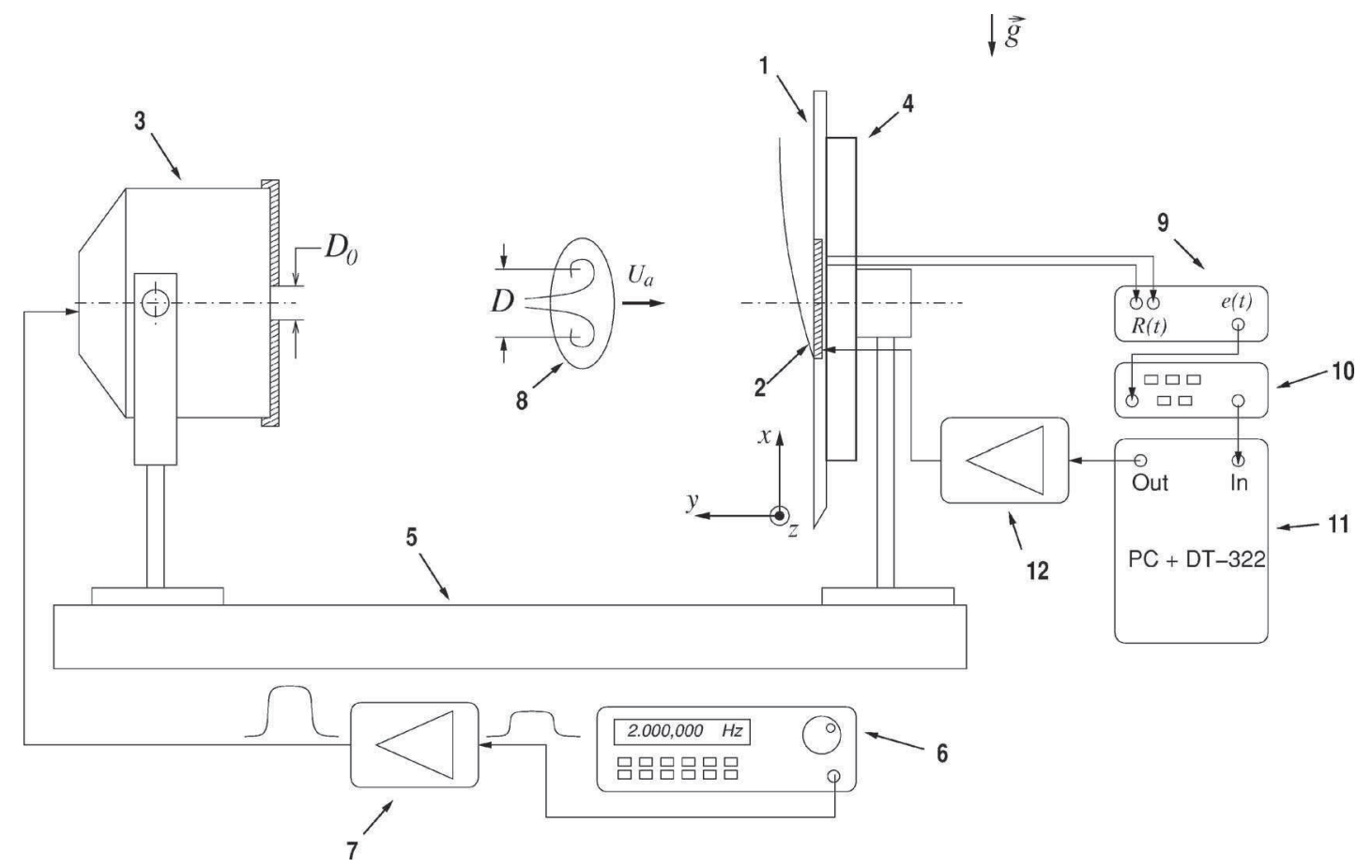

FIG. 1. (a) Sketch of the experimental setup. A plane wall (1) made of a $10 \times 20 \mathrm{~cm}$ and $5 \mathrm{~mm}$ thick plexiglass plate supports a thin resistive heater film (2) placed at the center of the wall and facing the vortex ring generator (3). To avoid undesired heat losses, a styrofoam plate (4) was placed behind the wall. Both the generator and the wall were mounted over an optic rail (5) for proper alignment. An arbitrary waveform generator HP33120A (6) sends a driving signal to the vortex generator, previously amplified by an HSA 4011 high-speed power amplifier (7). This way, a known vortex ring is generated (with very good repeatability) (8), moving toward the heater plate maintained at constant temperature $T_{w}$. To do that, a Wheatstone bridge (9) reads the heater's resistance $R$. The bridge error $e(t)$ is amplified with a SR-560 preamplifier (10) and then digitized with a DT-322 A/D system (11) at a sampling rate of $100 \mathrm{~Hz}$. A software PI controller computes the corrected output, which is amplified with a follower emitter circuit (12) and sent to the Wheatstone bridge in order to close the control loop and bring back the bridge's balance.

signal is received from the acquisition card (more details can be found in Ref. 10). The just created ring approaches a vertical flat heated plate centered in the axis of the ring at a distance of $20 \mathrm{~cm}$ from the ring generator. The heated plate is made of a $10 \mu \mathrm{m}$ thick Cermet film, deposited by serigraphy on a Kyocera substrate and heated by the Joule effect. Two high conductivity metallic strips, deposited on both sides of the film, provide a homogeneous electrical current across the film, thus obtaining a constant surface temperature on the film. Temperature homogeneity of the film was tested by infrared thermography. Temperature differences on its surface were not detectable with an IR camera $\left(0.2{ }^{\circ} \mathrm{C}\right.$ sensitivity). The heated plate was maintained at constant temperature by electronic feedback. By measuring the dc component and power fluctuations necessary to maintain it at constant temperature, we can determine the thermal flux released into the air flow surrounding the plate, and therefore the heat transfer enhancement or gain produced by a single or multiple vortex ring collisions.

\section{B. Properties of produced vortex rings}

Vortex rings were previously characterized using thermal anemometry ${ }^{10}$ using a calibrated TSI hot-film probe $(50 \mu \mathrm{m}$ in diameter) operated with a constant temperature anemometer. Probe position $\left(x, y_{0}, z\right)$ was controlled by a two-axis stepper motor system with resolution of 0.48 and $0.49 \mathrm{~mm} / \mathrm{rev}$ in the $x$ and $z$ directions, respectively. The hotfilm velocity signal (absolute value) was lowpass filtered
(12 dB/Oct@ @ $300 \mathrm{~Hz}$ ), amplified with a SR-560 preamplifier, and then sampled by a DT-322 Data Translation $250 \mathrm{kHz}$ and 16 bits acquisition card. The forcing signal $s(t)$ was generated by a function synthetizer HP33120A, then amplified by a power amplifier HSA 4011. An external trigger signal starts both the ring emission and the A/D sampling.

Typical point measurements of the vortex ring velocity profile, with a single probe, were obtained as shown in Fig. 2(a) for three diameters $D_{0}=13,15,17 \mathrm{~mm}$ and different initial impulses. They display a characteristic high velocity central zone bounded by two sharp lateral minima (the hot film probe delivers the absolute value of flow velocity) followed by a $\sim x^{-1}$ decreasing region (irrotational region). The two lateral minima correspond to the centers of rotation that we observe in the image of Fig. 2(b), where we show a flow visualization of a starting ring made with a laser light sheet and smoke as a tracer. We illuminated an $(x, y)$ midplane and then we recorded these images with a CCD camera. This stable velocity profile is very similar to those obtained for thick-cored rings found in Ref. 11, where an estimation of the vortex core using their method gives us a ratio of the order of $a / R \sim 0.5$, indicating that we are working with thick rings.

In order to get a complete velocity profile with a single hot-film probe, the procedure is as follows: (i) we create a single ring (realization), (ii) perform the velocity measurement at an initial spatial position $\left(x_{0}, y_{0}, z_{0}\right)$, (iii) wait a cer- 


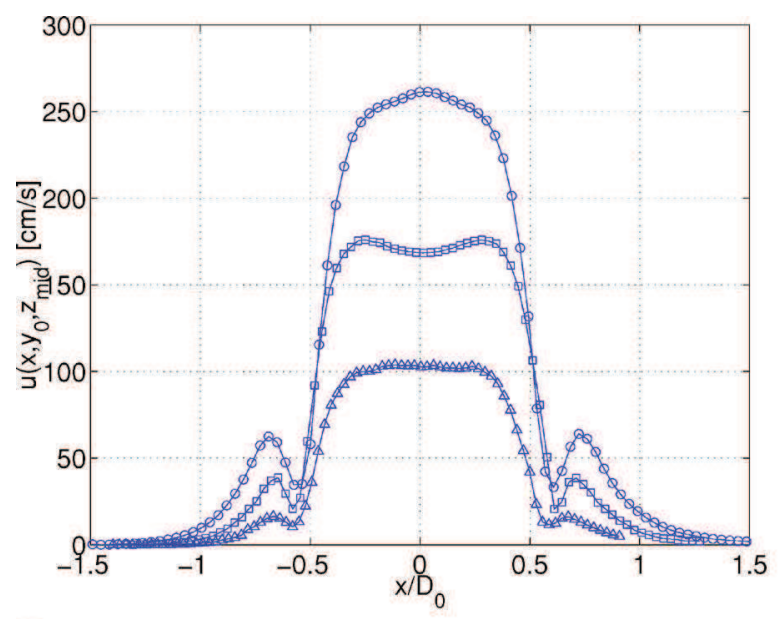

(a)
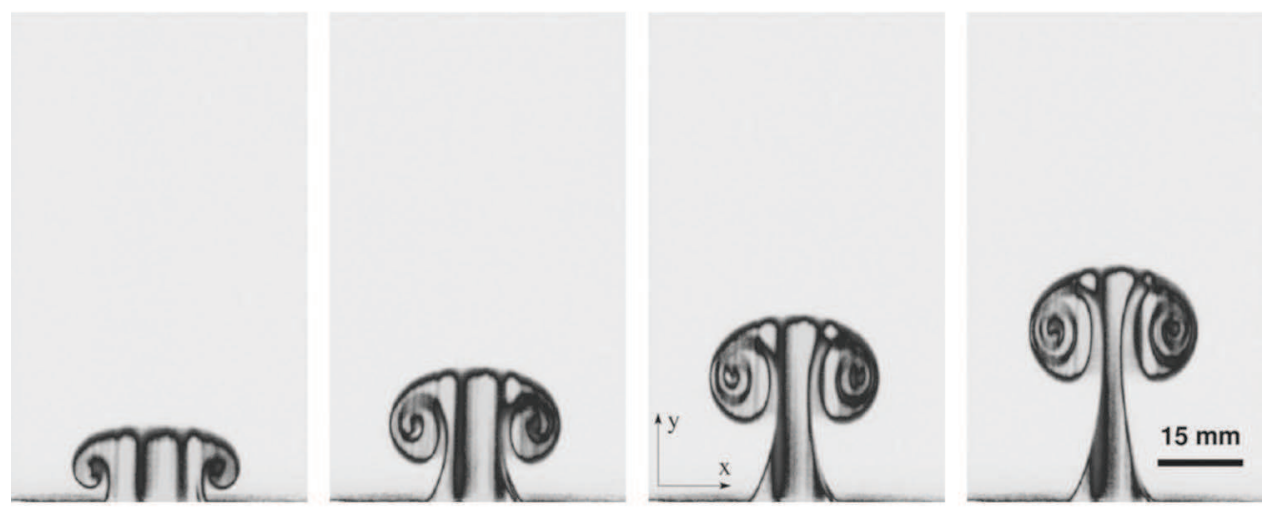

FIG. 2. (Color online) (a) Velocity profile for $D_{0}=13 \mathrm{~mm}$ at $y_{0} / D_{0}$ $=70 / 13, \quad \tau_{r}=15.1 \mathrm{~ms} \quad(\bigcirc) ; \quad D_{0}$ $=15 \mathrm{~mm}$ at $y_{0} / D_{0}=100 / 15, \quad \tau_{r}$ $=15.1 \mathrm{~ms}(\square) ; D_{0}=17 \mathrm{~mm}$ at $y_{0} / D_{0}$ $=150 / 17, \tau_{r}=11.2 \mathrm{~ms}(\triangle)$. (b) A midplane $(x, y)$ visualization of a starting ring for $D_{0}=15 \mathrm{~mm}, \tau_{r}=35.4 \mathrm{~ms}$. Observe the ring's wake structure and dimensions. After a few milliseconds, a wake pinch-off occurs leaving the vortex ring with a thinner wake.

(b)

tain time (many seconds) until the perturbation associated with the vortex ring wake vanishes, and (iv) then we must change the probe $(y, z)$ position without misalignments to start again. This procedure can be repeated automatically to make a complete scan in the $x$ coordinate. The stability of ring production was tested on the basis of successive measurements of flow velocity at different spatial locations, previously programmed on our stepper motor system. Velocity fluctuations of the order of $2 \%$ were found between measurements performed one day and repeated after one week.

Typical hot-film velocity measurements were described in detail in Ref. 10, where we recorded the evolution of basic properties of the vortex ring, such as its strength or circulation $\Gamma$, ring size $D=2 R$ during its motion, self-induced velocity $U_{a}$, and an estimation of the core size of the vortex ring. In this work, we will consider the average self-induced velocity of the ring $U_{a}$ and the diameter $D_{0}$ as the varying parameters to evaluate the interaction between the ring and the vertical boundary layer. Thus, the governing dimensionless parameter is the Reynolds number, defined as $\operatorname{Re}=U_{a} D_{0} / \nu$

\section{Properties of the natural convection boundary layer}

Typical properties of the boundary layer were determined before the ring interaction. The heated plate (dimensions $H=5.08, W=5.08 \mathrm{~cm}$ ) is made of a resistive thin me- tallic oxide (Cermet) film $10 \mu \mathrm{m}$ thick, deposited in a ceramic substrate and heated by the Joule effect at constant temperature $T_{w}$ with a digital feedback PI controller. The heated plate was bounded by a larger plexiglass smooth plate (size $10 \times 20 \mathrm{~cm}$ ) which was back-insulated with a plate of Styrofoam of $20 \mathrm{~mm}$ width as indicated in Fig. 1. Between the heater plate and back-insulation, a temperature sensor (LM35DZ, accuracy $\pm 0.5^{\circ} \mathrm{C}$ ) was mounted flush in order to have an average center temperature of the plate in the stationary regime.

The digital PI heater controller has a flat frequency response up to $50 \mathrm{~Hz}$, therefore allowing to sense fast and small power fluctuations of the boundary layer associated with ring collisions with an accuracy of $\pm 1 \mathrm{~mW}$. It maintains the heater resistance $R$ constant, thus a constant wall temperature $T_{w}$, with a uniformity of $0.5{ }^{\circ} \mathrm{C}$ and stability better than $0.05{ }^{\circ} \mathrm{C}$. Room-temperature stability is of the order of $0.5{ }^{\circ} \mathrm{C}$ during $24 \mathrm{~h}$.

Local temperature profiles of the boundary layer were measured with a small NTC thermistor $(0.5 \mathrm{~mm}$ in diameter and $10 \mathrm{~s}$ of rising time) mounted on a two-axis stepper motor system and sampled at $64 \mathrm{~Hz}$ with the DT322 A/D card. Once the heated plate reached an operating and constant temperature $T_{w}=50{ }^{\circ} \mathrm{C}$, temperature profiles $T(y)$ were obtained automatically from the heated plate moving the temperature sensor along the normal and vertical coordinates with increments of $\delta y=0.4 \mathrm{~mm}$ and $\delta x=10 \mathrm{~mm}$, respectively. 


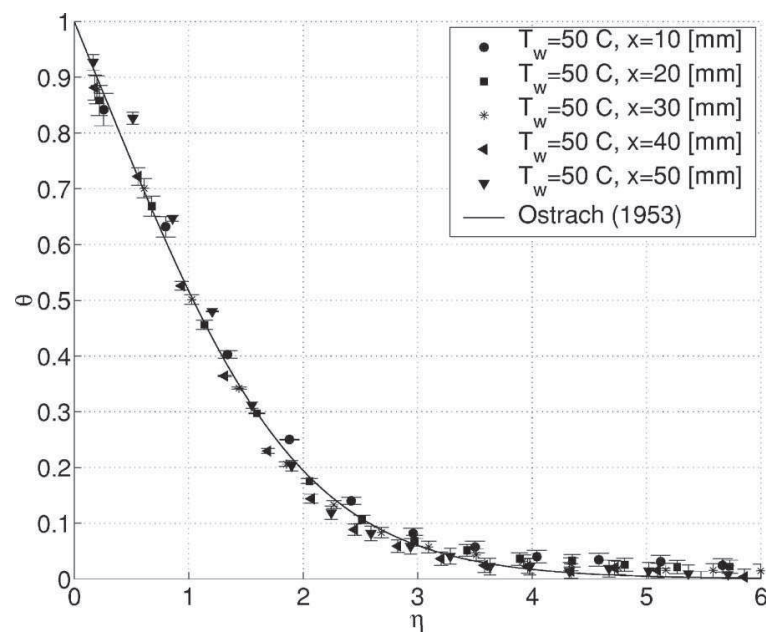

FIG. 3. Dimensionless temperature profiles $\theta(\eta)$ for several values of the $x$ coordinate along the plate. Results show an adequate curve collapse for $0<\eta<4$ (even though we are dealing with a finite-size heated plate) in agreement with the standard scaling (solid line) found in many textbooks (see, for instance, Ref. 12). All measurements shown were performed at $T_{w}=50{ }^{\circ} \mathrm{C}$.

In order to check self-similarity of the boundary layer temperature profile, dimensionless temperature $\theta$ was plotted versus a dimensionless coordinate $\eta$ involving the Grashof number $G_{x}$ and defined as

$$
\begin{aligned}
& \theta=\frac{T-T_{\infty}}{T_{w}-T_{\infty}}, \quad \eta=\frac{y}{x}\left(\frac{G_{x}}{4}\right)^{1 / 4}, \\
& G_{x}=\frac{g x^{3}\left(T_{w}-T_{\infty}\right)}{\nu^{2} T_{\infty}}, \quad \Delta T=\left(T_{w}-T_{\infty}\right),
\end{aligned}
$$

where $T_{\infty}, \nu$, and $g$ are the room temperature, kinematic viscosity of air, and gravitational acceleration, respectively. Figure 3 shows the temperature profiles for different vertical positions along the heated plate. A fairly good collapse is found using the dimensionless parameters described above, which is in good agreement with the standard scaling (see, for instance, Ref. 12). Such a typical characterization is con-

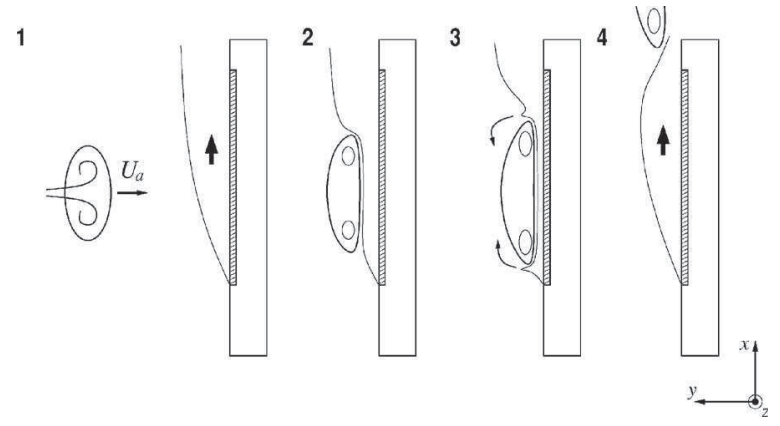

FIG. 4. Temporal sequence of boundary layer perturbation occurring by the impinging vortex ring. (1) Vortex ring approaching the heated plate. (2) Once the vortex ring gets closer to the wall, the boundary layer begins to be perturbed, increasing temperature and velocity gradients. (3) After that, boundary layer separation and entrainment of hot fluid into the vortex ring occurs. (4) Both the remanent ring and the perturbed boundary layer are convected away from the heater, and the natural convection regime is restored. (a)

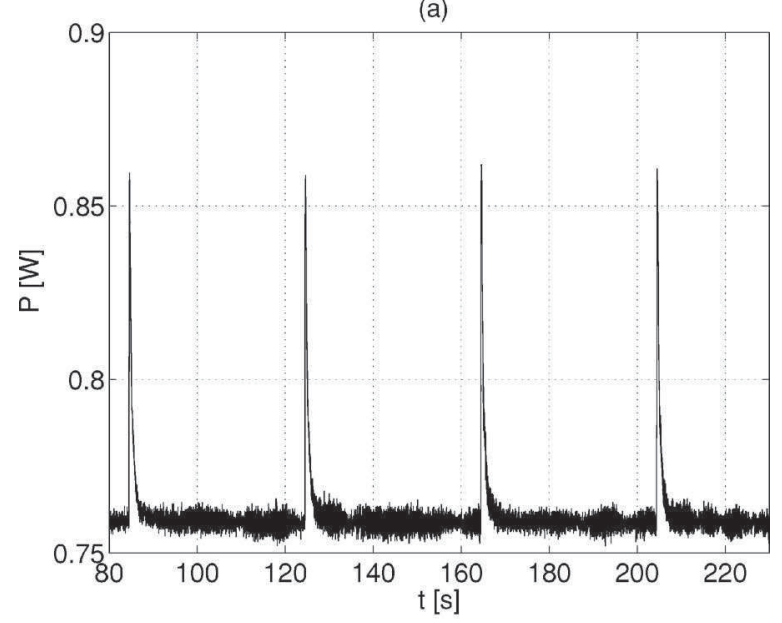

(b)

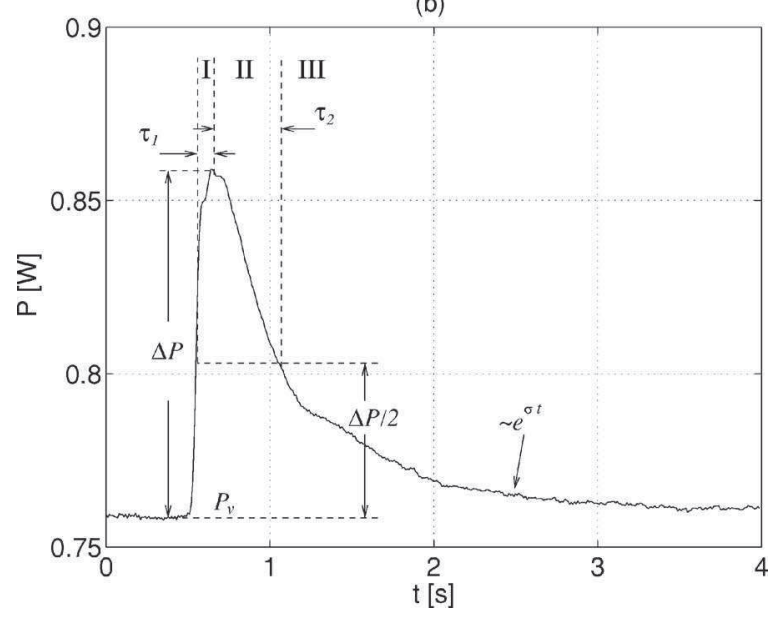

FIG. 5. Temporal evolution of the electrical heater power consumption (under PI feedback control at $T_{w}=50{ }^{\circ} \mathrm{C}$ ) originated by the vortex ring collision with the boundary layer. (a) A time series of four consecutive events sampled at $100 \mathrm{~Hz}$. (b) These events are then ensemble-averaged, obtaining a representative and detailed power evolution of the unsteady heat transfer process, where three time scales can be recognized $\left(\tau_{1}, \tau_{2}, \tau_{3}\right)$.

sidered important in this work prior to the study of ring impingement on the stationary boundary layer created along this vertical heated plate of finite size and maintained at constant temperature. Such a boundary layer permits us to estimate the heat flux in the form of

$$
q=H k N_{m}\left(T_{w}-T_{\infty}\right),
$$

where $H$ is the heater plate length and $k=0.0262 \mathrm{~W} / \mathrm{mK}$ is the molecular thermal conductivity of air at room temperature. ${ }^{13}$ Therefore, the mean Nusselt number $N_{m}$ can be written as $N_{m}=0.478 \cdot G^{1 / 4}$, where the Grashof number here is $G=g \cdot H^{3}\left(T_{w}-T_{\infty}\right) /\left(\nu^{2} \cdot T_{\infty}\right)$.

According to the previous definitions, the thermal boundary layer thickness $\delta_{0}$ verifies $H \cdot k N_{m} \Delta T$ $\sim H^{2} \cdot k \Delta T / \delta_{0}$. Therefore, $\delta_{0}$ can be expressed in terms of the Grashof number as $\delta_{0} \sim H G^{-1 / 4}$. In the present work, the Grashof number reaches a maximum value of $10^{5}$, which indicates that the natural convection flow is always laminar.

This natural convection regime has an associated dynamic boundary layer, and since the Prandtl number $\operatorname{Pr} \sim 1$, both thermal and dynamic boundary layers have similar char- 
(a)

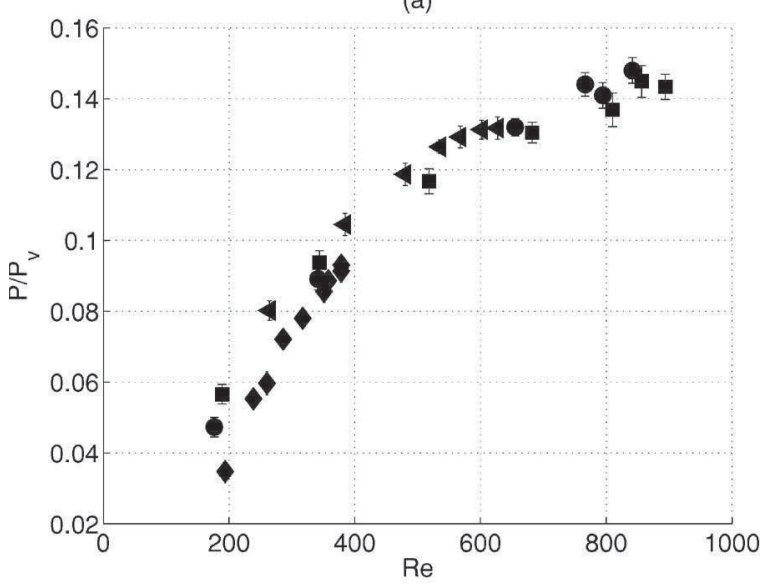

(b)

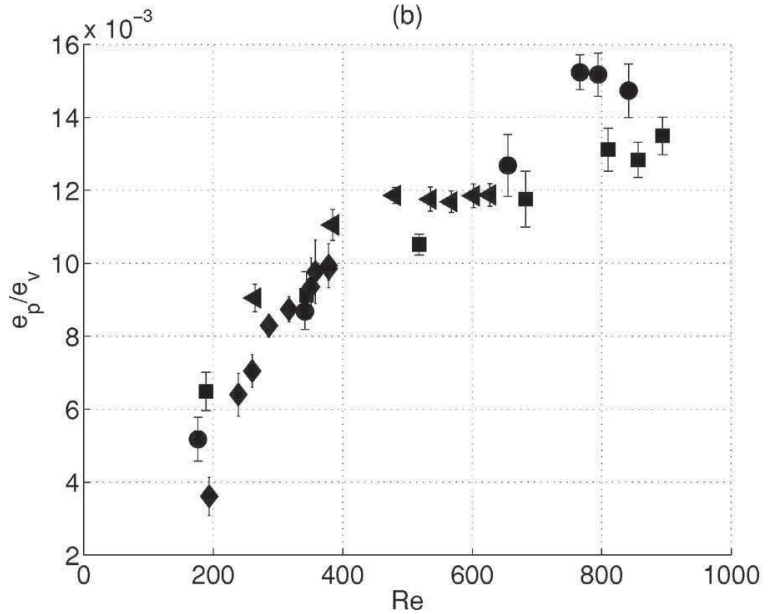

(c)

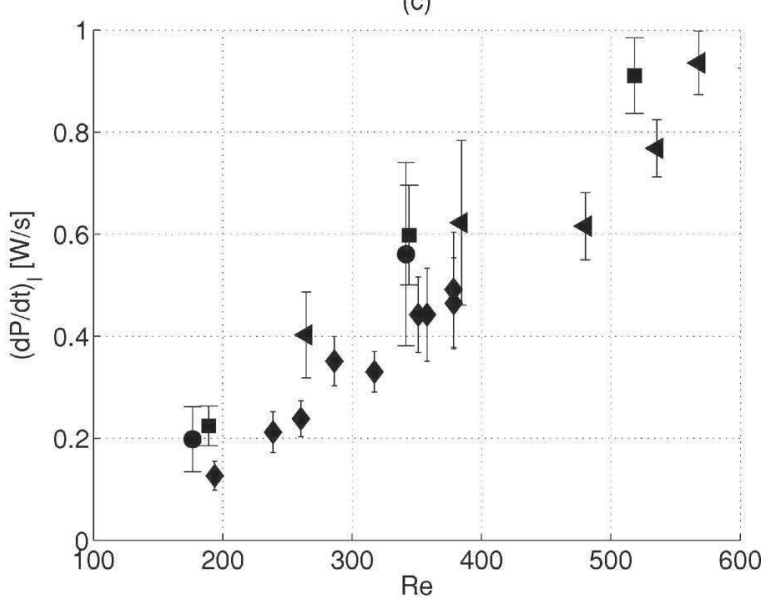

(d)

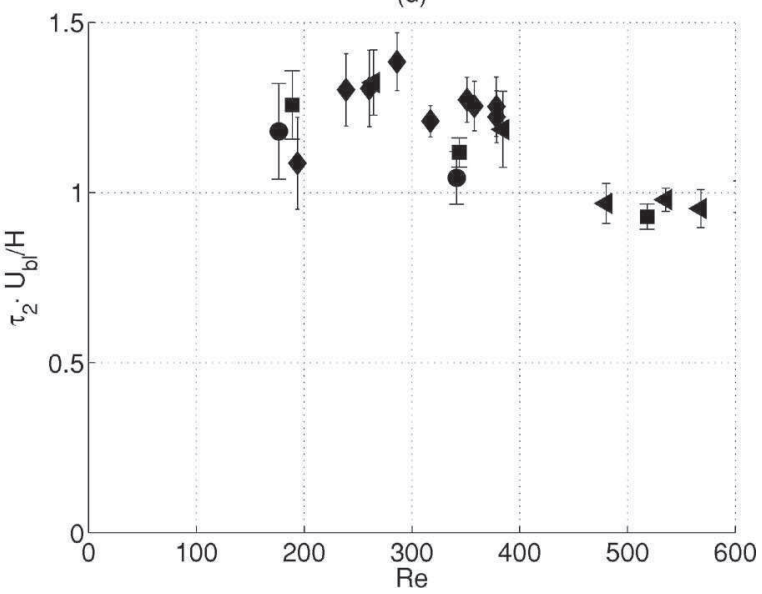

FIG. 6. (a) Dimensionless power gain $P / P_{v}$, where $P_{v}$ is the unperturbed heat flux (without impinging vortex ring) for our vertical heated plate. (b) Dimensionless dissipated energy $e_{p} / e_{v}$, where $e_{v}$ corresponds also to the dissipated energy by the heater in absence of impact. (c) Time derivative of the power curve at zone I vs Reynolds number Re. (d) Dimensionless time $\tau_{2}$ vs Reynolds number Re. $(\bullet) D_{0}=13 \mathrm{~mm} ;(\boldsymbol{\square}) D_{0}=15 \mathrm{~mm} ;(\varangle) D_{0}=17 \mathrm{~mm} ;(\bullet)$ $D_{0}=20 \mathrm{~mm}$.

acteristic scales. ${ }^{14}$ The dynamic boundary layer has a selfsimilar velocity profile, with a characteristic velocity which obeys to $U_{\mathrm{bl}} \sim \sqrt{g H \Delta T / T_{\infty}} \cdot 12$

The conduction heat losses through the system can be obtained by comparison with the electric power delivered by the feedback controller to the heated plate. The heat flux is obtained from the controller output such that

$$
q_{w}=I^{2} \frac{R}{A}-q_{c}
$$

where $I$ is the electric current in the heating film, $R$ is the overall electric resistance of the heating film, $A$ is the surface or total heater area $\left(A=H^{2}\right)$, and $q_{c}$ is the conduction loss through the insulation material. Note that radiation heat losses can be neglected since the present experiment is conducted with a surface temperature $T_{w}=50{ }^{\circ} \mathrm{C}$. In our experiments, heat losses were minimized mounting the heated plate into the plexiglass wall with a small air gap in between. Heat losses by conduction to the plate support represent a $\sim 15 \%$ of the steady-state ground power, $P_{v}$, shown in Fig. 5. Heat losses by conductive transients in the support and thermal insulation take place only when the system starts, and it takes several minutes until it reaches a stationary regime. Thus these transients are too slow to be taken into account for the power fluctuations associated with the ring impingement process.

\section{RING IMPINGEMENT AND HEAT TRANSFER}

A scheme of the ring interaction with the boundary layer is illustrated in Fig. 4 based on flow visualizations. A single ring approaches the boundary layer with an average selfinduced velocity $U_{a}$, estimated as $U_{a}=L / \tau$, where $L$ is the distance between the ring generator and the heated plate; $\tau$ is the time lag between the generation and the impingement process. Once the vortex ring gets closer to the wall, both boundary layers (thermal and dynamic) begin to be perturbed, increasing local temperature gradients. The circular ring shape is almost preserved during a short time in front of the heated plate. After that, entrainment of hot fluid into the vortex ring begins to take place. Then both the perturbed boundary layer and the ring are convected upstream from the heater plate, and after a characteristic time, the natural convection regime is restored. All characteristic times of the 
collision can be observed in the plot of power fluctuations delivered by the feedback controller (Fig. 5). The thermal unsteady response of the boundary layer during ring impingement is illustrated in Fig. 5. Once the heated vertical plate reaches a constant temperature $T_{w}=50{ }^{\circ} \mathrm{C}$, which is maintained by the PI feedback controller, we record the instantaneous electrical power dissipated by the heater [see Eq. (3)]. When the system is in steady state, the background power dissipated by the boundary layer reaches a constant value $P_{v}$. If we send a single vortex ring to the wall across the boundary layer, we get a positive peak of electric power corresponding to a heat transfer enhancement originated by the ring impingement on the heated plate. This procedure can be repeated several times to get a characteristic average of this unsteady heat transfer process, increasing signal-to-noise ratio. We have plotted the temporal evolution of the heater power consumption during feedback control originated by the vortex ring impingement. The time series of Fig. 5(a) represent four consecutive vortex ring collisions with the heated plate. Such events are then ensemble-averaged (as we know the time origin for each collision) obtaining a characteristic power evolution such as that of Fig. 5(b).

The power signal reveals different stages in the collision process as illustrated in Fig. 5(b). We recognized three time scales involved. The first time scale $\tau_{1}$ is the rising time of the power fluctuation (region I). This time scale is defined by the ratio between the boundary layer thickness and the ring velocity, $\tau_{1} \sim \delta_{0} / U_{a}$. The second time scale, $\tau_{2}$, is the settling time of the power fluctuation curve (region II). In this stage, an almost linear decay of dissipated power can be observed, which is related to the advection of the vortex ring by the dynamic boundary layer. Thus, this time scale is defined by the ratio between the size of the vertical heated plate and the convective velocity of the boundary layer, $\tau_{2}=H / U_{\mathrm{bl}}$. Indeed, this time delay is not significantly dependent on the Reynolds number of the incoming ring, as shown in Fig. 6(d). After the first part of vortex ring impingement $\left(t \sim \tau_{1}\right)$, the remanent of the vortex ring is convected away $\left(t \sim \tau_{2}\right)$ leaving the heated plate, and then the boundary layer begins to be restored $\left(t \sim \tau_{3}\right)$. This last time scale, written as $\tau_{3} \sim \delta_{0}^{2} / \nu$, corresponds to the time required by the boundary layer to return to its unperturbed initial state. Our measurements confirm that $\tau_{3}$ does not significantly vary with the range of $\Delta T$ considered here, and $\tau_{3} \sim 0.8 \mathrm{~s}$.

The transient response of the dissipated power demonstrates that vortex ring impingement can be considered as a heat transfer enhancement method. The amount of extra heat released during the ring collision can be manipulated introducing changes in the vortex ring properties, such that the self-induced velocity $U_{a}$.

The power signal of Fig. 5(b) is the signature of the ring collision in terms of heat transfer. From this signal, the power peak height, defined just above the background constant power $P_{v}$, is considered here as the characteristic power gain of the process. Also, the power curve $P(t)$ can be integrated over time in order to obtain a measure of the thermal energy released by ring impingement, $e_{p}=\int_{t_{0}}^{t_{0}+\Delta t}\left[P(t)-P_{v}\right] \mathrm{d} t$. In the previous definition of $e_{p}$, the power curve was integrated in a constant time interval $\Delta t$, which takes into ac- (a)

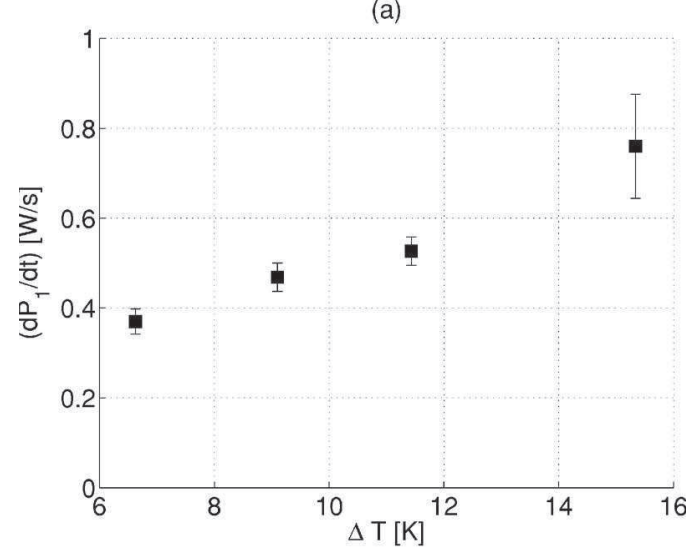

(b)

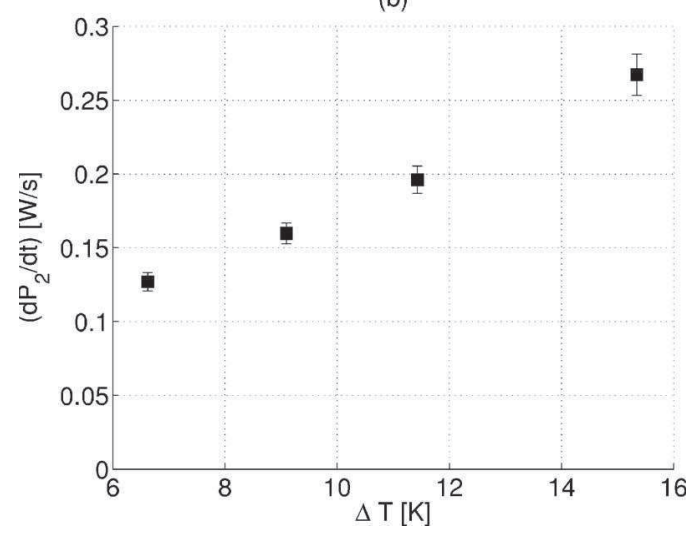

FIG. 7. Rising slope (a) and settling slope (b) at regions I and II for different heater temperatures $T_{w}$ and a constant surrounding temperature $T_{\infty}$, giving different values for $\Delta T$. In this experiment, the vortex rings were generated with $D_{0}=17 \mathrm{~mm}, \tau_{r}=7.7 \mathrm{~ms}$, where the Reynolds number is $\operatorname{Re} \sim 600$. These results are in agreement with the fact that thermal boundary layer thickness decreases and the dynamic boundary layer velocity increases as $\Delta T$ increases.

count all the power transients originated by the impingement process. With these definitions, the power gain $\Delta P$ and energy $e_{p}$ were recorded in order to classify the effect of vortex rings of different size $D_{0}$ and self-induced velocity $U_{a}$. Both the power gain $\Delta P$ and the dissipated energy $e_{p}$ were found to increase with the ring Reynolds number $\operatorname{Re}=U_{a} D_{0} / \nu$ as shown in Figs. 6(a) and 6(b). Note that the error bars on the figure do not represent the accuracy of the measurement, but the standard deviation on a set of experiments that are ensemble averaged. In addition to the $\Delta P$ and $e_{p}$ versus $\mathrm{Re}$ plots, we compute the time derivatives of heat flux at the regions I and II as a function of the Reynolds number, as shown in Figs. 6(c) and 6(d). It can be observed that both dimensionless time derivatives at regions I and II increase linearly with the Reynolds number. Since the rising time $\tau_{1}$ depends on the inverse of $U_{a}$, it can be expected that dimensionless time derivative at region I grows with Re at the early stages of vortex ring/heater interaction.

The evolution of these time derivatives with $\Delta T$ was also considered. In order to do that, at constant surrounding temperature $T_{\infty}$, different values of $T_{w}$ were considered. Figure 7 shows the results obtained for $6<\Delta T<16{ }^{\circ} \mathrm{C}$. It can be observed that time derivatives for regions I and II increase as 


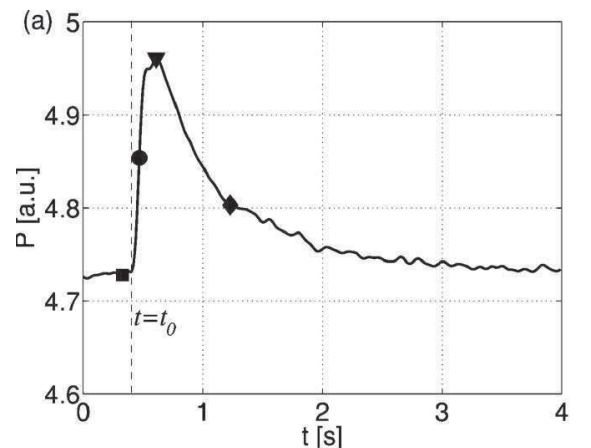

(c)

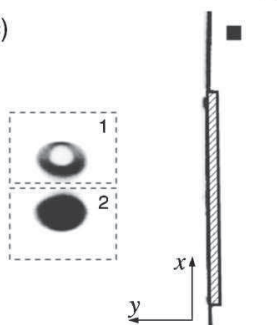

(d)

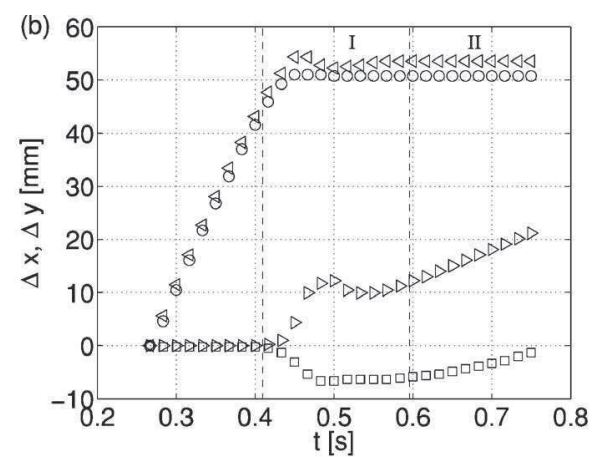

(e)

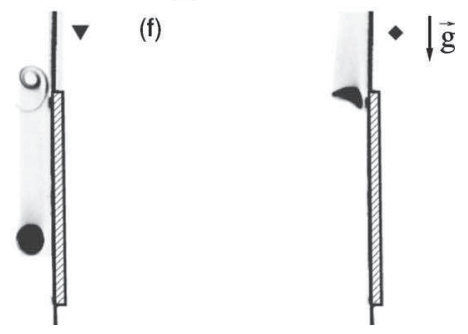

FIG. 8. Synchronized flow visualization and power data measurements. (a) Power fluctuations during the impingement process where four time instants are indicated by symbols for flow visualization: ( $\boldsymbol{\square})$ just before the impact, ( $)$ at half-maximum peak, ( $)$ at maximum peak, and ( ) between regions II and III. (c,d) Flow visualization for each time instant. In (c), top and bottom interrogation windows are shown, which are used to follow the current position of the upper and lower portions of the ring with time, using a PIV algorithm. These relative positions are plotted in (b), where ( $\triangleleft$, $\triangleright)$ correspond to the horizontal and vertical position for the upper portion and $(\bigcirc, \square)$ for the lower portion, respectively. It is observed that (1) at the early stages of the impact $(\square$ and $\boldsymbol{\bullet})$, the boundary layer has not been perturbed enough to increase the transfer rate and the ring diameter remains close to its initial size; (2) when the power reaches its maximum value, the ring's diameter increases considerably $(\boldsymbol{\nabla})$, thus increasing the heat transfer area; (3) as the ring is convected away from the heater, dissipated power decreases almost linearly (between $\boldsymbol{\nabla}$ and $\downarrow$ ); (4) after $\downarrow$ the ring has left the heater and the boundary layer begins to be restored.

$\Delta T$ increases. The former can be explained on the basis of the relationship $\delta_{0}=H G^{-1 / 4}$, so $\delta_{0}$ decreases as $\Delta T$ increases and thus $\tau_{1}$ decreases consequently. The latter occurs due to the well known increase of the dynamic boundary layer velocity with $\Delta T$. $^{14}$

To better understand the measurements done on the average heat flux signal and to clarify the mechanism underlying the impingement process, we performed flow visualiza- tions, using a laser sheet and smoke as a tracer, on typical single vortex ring realizations.

Typical lateral views are shown in Fig. 8, where the laser sheet was placed vertically to obtain a midplane view of the ring during its trip to the heated plate. From Fig. 8, it is possible to follow the current position of the ring by computing the $2 \mathrm{D}$ correlation between two consecutive frames $(A(x, y, t), B(x, y, t+\Delta t))$ computed as (a)

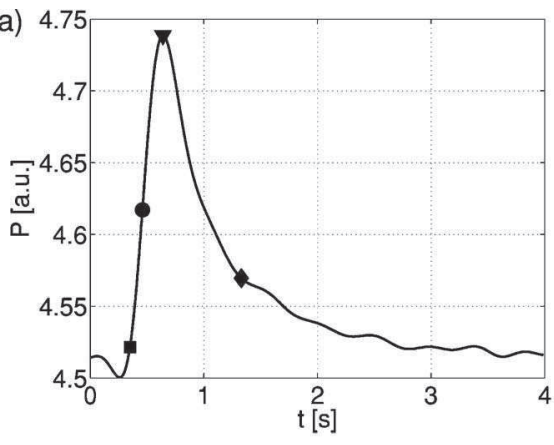

(c)

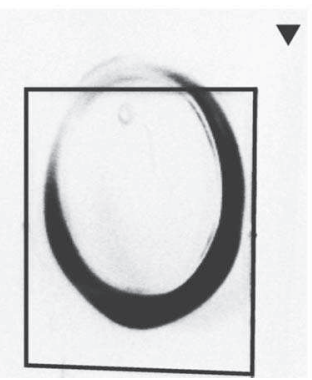

(b)

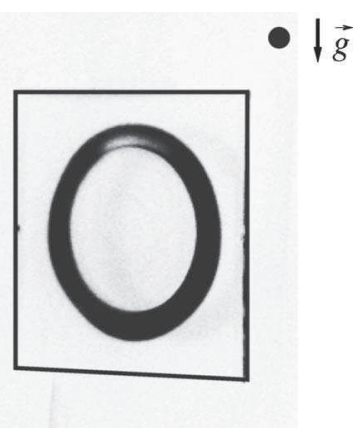

(d)

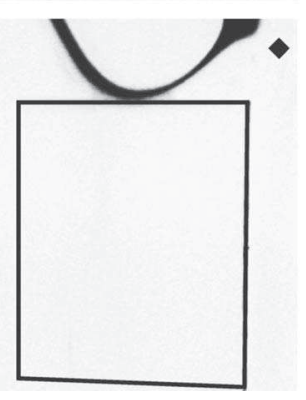

FIG. 9. Front view visualization for $D_{0}=13$, $\tau_{r}=35.4 \mathrm{~ms}, U_{a}=39.43 \mathrm{~cm} / \mathrm{s}$. An asymmetric growth of the ring can be observed ( $\boldsymbol{\nabla})$, as resulting from the interaction between the ring and the dynamic boundary layer generated by the heater. Since the sign of vorticity at the lower region of the ring has the opposite sign of the upper region, the wall vorticity increment generated by the ring impingement is not symmetric, thus originating a slower expansion at the bottom of the ring. 


$$
C_{A, B}(x, y)=\sum_{i=1}^{M} \sum_{j=1}^{N} A(i, j, t) \cdot B(x-i, y-j, t+\Delta t)
$$

using Fourier transform methods according to the correlation theorem. $M$ and $N$ are the dimensions of the interrogation area (in pixels). To compute the spatial position $(\Delta x(t), \Delta y(t))$ of the vortex ring, we define an interrogation area that includes a side view around the vortex core [see Fig. 8(c)]. After that, we use the Fourier version of Eq. (4) to find out the cross-correlation maximum between consecutive frames to obtain relative positions in time of the vortex ring and therefore the overall displacements in a given time.

It is observed that at the early stages of the impact [Fig. $8(c)$, neither the boundary layer nor the vortex ring are perturbed enough to see an increase of the heat transfer. This is confirmed in Fig. 8(b), where the vortex ring position $(\Delta x(t), \Delta y(t))$ is shown at four time instants. The $y$ position of the ring increases linearly in time just before the impact, showing that the self-induced velocity remains nearly constant. However, the $x$ position remains constant just before the impact, indicating that the ring's diameter does not change.

At $t=t_{0} \quad$ [see Fig. 8(a)], the vortex ring position $(\Delta x(t), \Delta y(t))$ changes abruptly, thus indicating the beginning of the impingement process. The $\Delta y(t)$ position for $t>t_{0}$ remains constant with time as the vortex ring is stopped by the wall and the ring's diameter grows rapidly, increasing the heat transfer area, as shown by the $\Delta x(t)$ curve. Simultaneously [Fig. 8(a)], the heat flux reaches its maximum value. After that, the ring is convected away from the heater, the dissipated power decreases almost linearly, and when the ring has quit the heater, the boundary layer begins to be restored and the power signal approaches the stationary power value.

It is interesting to note that after impingement, the vortex is advected by the boundary layer flow, but the vertical velocities corresponding to the top and bottom side views are different. This can be explained by the sign of local vorticity of the ring ${ }^{7,15}$ and that of the dynamic boundary layer. Front view visualizations during the impingement (Fig. 9) confirm an asymmetric ring development, where the upper ring portion goes faster than the lower portion, and therefore the ring expands faster along the vertical direction.

From these results, it has been possible to reveal a dual mechanism, with different time scales, involved in the heat transfer enhancement during ring impingement. A first part, identified with the ring expansion, ends when the ring diameter stops growing (saturated diameter). The second part, characterized by the vertical advection of the vortex ring, ends when the ring quits the heated plate.

\section{CONCLUSION AND PERSPECTIVES}

The impact of vortex rings on a vertical heated plate, its dynamical behavior, and the consequences on heat transfer were studied in our experiments.
Different tests we carried out show the following. (i) In the stationary regime, the heat flux remains nearly constant and a laminar convective boundary layer is generated in the flow surrounding the wall. (ii) When a vortex ring impacts the heated plate, a sudden change of the heat flux occurs, in the form of a positive peak of power that vanishes when the ring is convected upstream the heated plate. Since the heated plate temperature was maintained constant, this power peak can only be explained by a localized heat transfer enhancement originated by the ring impingement process, where a dual mechanism explains the unsteady heat transfer peak. (iii) Different vortex rings were generated, and it was found that thermal energy released increases when the ring's Reynolds number increases. (iv) Synchronized flow visualizations of the impingement process allow us to conclude that the mechanism increases mixing and distortion of the unperturbed boundary layer generated by the heater, resulting in an enhancement of the heat transfer process. (v) The order of magnitude of the kinetic energy of the vortex ring is $e_{c} \sim 10^{-6}(\mathrm{~J})$ just before impact, and the amount of additional thermal energy $e_{p}$ released during the impingement process is of the order of $e_{p} \sim 0.1(\mathrm{~J})$, therefore $e_{p} \gg e_{c}$. This ratio provides some guidelines about the efficiency of this process.

As the impingement process is very localized in time, it is possible to generate periodic series of vortex rings in order to further increase the heat flux, improving the efficiency of this heat transfer enhancement method. The absence of undesired acoustic noise, the low energy consumption, and the ability to aim the ring in any direction make this method attractive in some applications involving zero-gravity conditions (work under progress) and at smaller scales as in the framework of microfluidics and MEMS.

\section{ACKNOWLEDGMENTS}

Support from Fondecyt Grants No. 1020134 and No. 7020134 is gratefully acknowledged. F.P. is happy to thank Facultad de Ciencias Físicas y Matemáticas of Universidad de Chile for kind hospitality. We acknowledge and thank CETHIL lab for the lending of the IR camera.

\footnotetext{
${ }^{1}$ A. Jacobi and R. Shah, "Heat transfer surfaces enhancement through the use of longitudinal vortices: A review of recent progress," Exp. Therm. Fluid Sci. 11, 295 (1995).

${ }^{2}$ K. Shariff and A. Leonard "Vortex rings," Annu. Rev. Fluid Mech. 24, 235 (1992).

${ }^{3}$ P. R. Voke and S. Gao, "Numerical study of heat transfer from an impinging jet," Int. J. Heat Mass Transfer 41, 671 (1998).

${ }^{4}$ G. Charnay, J. Mathieu, and G. Comte-Bellot, "Response of a turbulent boundary layer to random fluctuations in the external stream," Phys. Fluids 19, 1261 (1976).

${ }^{5}$ A. Kondjoyan, F. Péneau, and H. Boisson, "Effect of high free stream turbulence on heat transfer between plates and air flows: A review of existing experimental results," Int. J. Therm. Sci. 41, 1 (2002).

${ }^{6}$ J. Walker and C. Smith, "The impact of a vortex ring on a wall," J. Fluid Mech. 181, 99 (1987).

${ }^{7}$ P. Orlandi and R. Verzicco, "Vortex rings impinging on walls: axisymmetric and three-dimensional simulations," J. Fluid Mech. 256, 615 (1993).

${ }^{8}$ D. Fabris and D. Liepmann, "Quantitative experimental and numerical investigation of a vortex ring impinging on a wall," Phys. Fluids 8, 2640 (1996).

${ }^{9}$ S. James and K. Madnia, "Direct numerical simulation of a laminar vortex ring," Phys. Fluids 8, 2400 (1996).
} 
${ }^{10}$ R. H. Hernández, B. Cibert, and C. Bechét, "Experiments with vortex rings in air," Europhys. Lett. 75, 743 (2006)

${ }^{11}$ J. P. Sullivan, S. E. Widnall, and S. Ezekiel, "Study of vortex rings using a laser Doppler velocimeter," AIAA J. 11, 1384 (1973).

${ }^{12}$ H. Schlichting, Boundary Layer Theory (McGraw-Hill, New York, 1968).
${ }^{13}$ D. Lide, CRC Handbook (CRC Press, New York, 2002).

${ }^{14}$ A. Bejan, Convection Heat Transfer, 3rd ed. (Wiley \& Sons, New York, 2004).

${ }^{15}$ C. Chu, C. Wang and C. Chang, "A vortex ring impinging on a solid plane surface-Vortex structure and surface force," Phys. Fluids 7, 1391 (1995). 